\title{
Processing of Biomedical Data with Machine Learning
}

\author{
Marat Bogdanov \\ Ufa State Aviation Technical University \\ M.Akmullah named after Bashkir State \\ Pedagogical University \\ Ufa City, Russia \\ bogdanov_marat@mail.ru \\ Irina Dumchikova \\ M.Akmullah named after Bashkir State \\ Pedagogical University \\ Ufa City, Russia \\ redfoxufa@gmail.com
}

\author{
Dajan Nasyrov \\ Ufa State Aviation Technical \\ University \\ Ufa City, Russia \\ 9373007333@mail.ru \\ Artur Samigullin \\ M.Akmullah named after Bashkir \\ State Pedagogical University \\ Ufa City, Russia \\ samigullin.art@gmail.com
}

\begin{abstract}
The paper is about processing of biomedical data. It were used 13 methods of machine learning (Naive Bayes classifier for multivariate Bernoulli models, A decision tree classifier, An extremely randomized tree classifier, Classifier implementing the $k$ nearest neighbors vote, Linear Discriminant Analysis, Linear Support Vector Classification, Logistic Regression, Nearest centroid classifier, A random forest classifier, Classifier using Ridge regression, Ridge classifier with built in cross validation, Gaussian Mixture Models, Support Vector Machines) and one method of deep learning (Multiplayer Perception). A discrete wavelet transform was used to extract of biometric features. Haar wavelets, Daubechi wavelets, Symlets, Coiflets, Biorthogonal, Reverse biorthogonal, Discrete Meyer (FIR Approximation) were used. The influence of Electrocardiorams (ECG) recording time on the accuracy of biometric identification and diagnosis of cardiovascular diseases was studied. It was found that the best methods of classification are: Multiplayer Perception, An extremely randomized tree classifier, Classifier implementing the $k$ nearest neighbors vote and Logistic Regression aka logit MaxEnt classifier. Wavelet family doesn't affect significantly on accuracy of recognition. With increasing registration time, accuracy increases .
\end{abstract}

Keywords-Machine Learning, Deep Learning, ECG, Biometric authentication, Cardiovascular diseases diagnosis

\section{INTRODUCTION}

The electrocardiograms allows us to measure various biomedical parameters such as the heart rate, the rhythm of heartbeats, heart abnormalities and perform the emotion recognition and biometric identification [1]. One of the major fields of using of ECG analysis is diagnosis of cardiovascular diseases. As reported by the World Health Organization, cardiovascular diseases are the main reason for deaths worldwide [1]. Many researchers have used ECG signals for emotion recognition in addition to many other signals such as the electroencephalogram, skin temperature, blood pressure, electromyogram, heart rate variability, cortisol levels, and thermal imaging features [1]. ECGs are also being used in the field of biometric identification.
Depending on the application, the analysis contains several steps, such as preprocessing, feature extraction, feature selection, feature transformation and classification. When preprocessing ECG recordings are cleaning from different types of noise and artifacts. In the preprocessing step, the goals are to reduce such noise and artifacts to determine the fiducial points $(\mathrm{P}, \mathrm{Q}, \mathrm{R}, \mathrm{S}, \mathrm{T}$ (P-onset, P-peaks, P-offset, QRS-onset, QRS-offset, T-onset, T-peaks and Toffset)) and to avoid amplitude and offset effects to compare signals from different patients [1-5]. The preprocessing stage uses a filtering block to delete artifact signals from an ECG signal $[1,6]$. Often, an ECG signal is initially bandpass filtered with different frequency ranges before analyzing it.

In the literature, various feature extraction techniques have been proposed to expose the distinctive information from ECG signals for different purposes, such as analysis and classification. Those features can be used individually or in combination with other features [1].

The use of morphological features is also possible in ECG analysis. In different studies [7], morphological features were used to diagnose ECG signals.

Frequency-based techniques are among the most popular feature extraction techniques for representing ECG signals for classification purposes. Because ECG signals are nonstationary objects it makes wavelets an effective tool for the analysis of ECG signals [8] and for frequency-based feature extraction, with its powerful time-frequency localization property [1]. The wavelet transformation is a linear transform that decomposes a signal into components that appear at different scales [9]. Time localization of the spectral components can be obtained by wavelet analysis, as this provides the time-frequency representation of the signal [1].

There are various methods of classification that have been utilized for ECG analysis and classification tasks such as artificial neural networks (ANNs), LDA, $\mathrm{k}$ nearest neighbor $(\mathrm{kNN})$, support vector machine (SVM), decision tree (DT), and Bayesian classifiers. 
Artificial neural networks is intended to solve both linear classification and non-linear classification problems with various network structures and learning algorithms [1].

Linear discriminant analysis goal is maximize the ratio of the between-class variance to the within-class variance, and it provides the highest possible discrimination between different classes. LDA is utilized in some of the recent ECG classification studies [10].k nearest neighbor has a wide usage in most of the pattern recognition problems and is also employed in some recent ECG classification studies [11].

Support vector machine is utilized in most of ECG classification studies [12]

Decision tree learning aims to map observations about an item to a conclusion. This conclusion can be either a possible target class label or a target value. Decision trees is used in some ECG classification studies [13]. In addition to common decision tree approaches, there are some more specific decision tree structures that are used frequently for ECG classification [1]. The Random Forest Tree is a type of ensemble classifier that uses many decision trees [1, 14]. In this approach, multiple decision trees are trained with subsets of training data. This approach uses a type of majority voting in which the output class label is assigned according to the number of votes from all the individual trees. This approach is also frequently used for ECG classification studies $[1,14]$.

Bayesian classifiers are the systems that are based on Bayes' decision theory. This theory is a fundamental statistical approach [15]. The idea behind these classifiers is that if the class is known, the values of the other features can be predicted. If the class is not known, then Bayes' rule can be used to predict the class label according to the given feature values. In Bayesian classifiers, probabilistic models of the features are built to predict the class label of a new sample. Bayesian classifiers, which are one of the widely used methods for pattern recognition problems, are utilized in most of the recent studies. The types of Bayesian classifiers utilized for ECG classification are the Bayesian network [1], naïve Bayes [1], and Bayes maximum likelihood classifier [1].

There are various application fields for ECG analysis and classification tasks. These fields can be grouped as disease classification, heartbeat type detection, biometric identification, and emotion recognition.

Disease classification using ECG is critical for correct diagnosis of the heart diseases, much effort has been made to analyze and classify ECG signals that belong to various heart problems. The aim of these efforts is the early detection of heart disease in general. Early detection can rescue the patient's life or prevent permanent damage to human organs $[1,16]$. The heart problem that most of the studies have focused on is arrhythmia.

The purpose of Heartbeat type detection is to separate different ECG beats from each other. This task is a part of ECG data analysis. There are various efforts that have concentrated on the separation of different types of ECG beats from one another. These efforts differ according to the used ECG beat types and applied detection approaches.
There are many studies in the literature about heartbeat type detection $[1,17]$.

The purpose of biometric identification is usually to increase security for any reason. There are various types of biometric information that can be extracted from humans, such as face, fingerprint, and retinal data. There are many recent studies related to ECG-based biometric identification in the literature $[1,18]$.

Emotion recognition is the one of the fundamental techniques of affective computing, which is the key technology for humanmachine interaction [1, 19]. Emotion can be recognized from facial expression, speech, physiological signals, and so on. Some examples of emotions are joy, anger, sadness, and pleasure. In this paper, ECGbased emotion recognition is only addressed. There is a limited number of recent studies related to ECG-based emotion recognition in the literature [1].

\section{MATERIALS AND METHODS}

The goal of the paper is finding the best combination of wavelet type used for feature extraction when biometrcic authentication and diagnoses of cardiovascular diseases and method of Machine Learning which gives the best accuracy. The second task is assessing affect of time of ECG recording on accuracy of reconition.

We used a samples of digitized electrocardiograms derived from the PTB database [20]. When doing preprocessing we used a wfdb python library [21] downloaded from www.physionet.org web site and biosppy python library [22]. We used wavelet discrete transformation for feature extraction with following wavelets: Haar wavelets, Daubechi wavelets (from db1 to db38), Symlets (from sym2 to sym20), Coiflets (from coif1 to coef17), Biorthogonal (from bior1.1 to bior6.8), Reverse biorthogonal wavelet (from rbio1.1 to rbio6.8) and Discrete Meyer (FIR Approximation). When classification we used following methods of classification: Multiplayer Artificial Neural Network, Naive Bayes classifier for multivariate Bernoulli models, A decision tree classifier, An extremely randomized tree classifier, Classifier implementing the $\mathrm{k}$ nearest neighbors vote, Linear Discriminant Analysis, Linear Support Vector Classification, Logistic Regression aka logit MaxEnt classifier, Nearest centroid classifier, A random forest classifier, Classifier using Ridge regression, Ridge classifier with built in cross validation, Gaussian Mixture Models, Support Vector Machines.

\section{RESULTS AND DISCUSSION}

Results of biometrical identification and diagnosis of cardiovascular diseases are shown on tables from 1 to 4 . ECG recognition time vary from 5 to 20 seconds.

As we can see from Tables 1-4. the best methods of classification are: Multiplayer Perception, An extremely randomized tree classifier, Classifier implementing the $\mathrm{k}$ nearest neighbors vote and Logistic Regression aka logit MaxEnt classifier. Wavelet family doesn't affect significantly on accuracy of recognition. With increasing registration time, accuracy increases. 
TABLE I

Results of biometrical identification/diagnosis of cardiovascular diseases. Registration time is 5 seconds.

\begin{tabular}{|c|c|c|c|c|c|c|}
\hline \multirow[t]{2}{*}{ Method } & \multicolumn{2}{|c|}{ Haar } & \multicolumn{2}{|c|}{ Daubechies } & \multicolumn{2}{|c|}{ Symlets } \\
\hline & Diagnosis & Identification & Diagnosis & Identification & Diagnosis & Identification \\
\hline Multiplayer Perception & 0.95 & 0.97 & 0.96 & 0.98 & 0.95 & 0.98 \\
\hline $\begin{array}{l}\text { Naive Bayes classifier for multivariate } \\
\text { Bernoulli models }\end{array}$ & 0.21 & 0.50 & 0.21 & 0.50 & 0.22 & 0.50 \\
\hline A decision tree classifier & 0.85 & 0.67 & 0.85 & 0.67 & 0.84 & 0.67 \\
\hline An extremely randomized tree classifier & 0.94 & 0.95 & 0.94 & 0.95 & 0.94 & 0.95 \\
\hline $\begin{array}{l}\text { Classifier implementing the } \mathrm{k} \text { nearest } \\
\text { neighbors vote }\end{array}$ & 0.97 & 0.94 & 0.97 & 0.94 & 0.97 & 0.94 \\
\hline Linear Discriminant Analysis & 0.73 & 0.89 & 0.73 & 0.89 & 0.73 & 0.89 \\
\hline Linear Support Vector Classification & 0.73 & 0.81 & 0.73 & 0.81 & 0.72 & 0.81 \\
\hline $\begin{array}{l}\text { Logistic Regression aka logit MaxEnt } \\
\text { classifier }\end{array}$ & 0.76 & 0.97 & 0.76 & 0.97 & 0.76 & 0.97 \\
\hline Nearest centroid classifier & 0.29 & 0.59 & 0.29 & 0.59 & 0.29 & 0.59 \\
\hline A random forest classifier & 0.71 & 0.12 & 0.71 & 0.12 & 0.71 & 0.10 \\
\hline Classifier using Ridge regression & 0.73 & 0.43 & 0.73 & 0.43 & 0.73 & 0.42 \\
\hline $\begin{array}{l}\text { Ridge classifier with built in cross } \\
\text { validation }\end{array}$ & 0.73 & 0.43 & 0.73 & 0.43 & 0.73 & 0.42 \\
\hline Gaussian Mixture Models & 0.24 & 0.60 & 0.24 & 0.60 & 0.25 & 0.60 \\
\hline Support Vector Machines & 0.86 & 0.83 & 0.86 & 0.83 & 0.86 & 0.83 \\
\hline
\end{tabular}


TABLE I (CONTINUE)

Results of biometrical identification/diagnosis of cardiovascular diseases. Registration time is 5 seconds.

\begin{tabular}{|c|c|c|c|c|c|c|c|c|}
\hline \multirow[t]{2}{*}{ Method } & \multicolumn{2}{|c|}{ Coiflets } & \multicolumn{2}{|c|}{ Biorthogonal } & \multicolumn{2}{|c|}{ Reverse biorthogonal } & \multicolumn{2}{|c|}{ Discrete Meyer } \\
\hline & Diagnosis & Identification & Diagnosis & Identification & Diagnosis & Identification & Diagnosis & Identification \\
\hline $\begin{array}{l}\text { Multiplayer } \\
\text { Perception }\end{array}$ & 0.96 & 0.98 & 0.94 & 0.98 & 0.94 & 0.98 & 0.91 & 0.97 \\
\hline $\begin{array}{l}\text { Naive Bayes } \\
\text { classifier for } \\
\text { multivariate } \\
\text { Bernoulli models }\end{array}$ & 0.21 & 0.50 & 0.21 & 0.50 & 0.21 & 0.50 & 0.19 & 0.48 \\
\hline $\begin{array}{l}\text { A decision tree } \\
\text { classifier }\end{array}$ & 0.85 & 0.67 & 0.85 & 0.67 & 0.85 & 0.67 & 0.84 & 0.68 \\
\hline $\begin{array}{l}\text { An extremely } \\
\text { randomized tree } \\
\text { classifier }\end{array}$ & 0.95 & 0.95 & 0.94 & 0.95 & 0.94 & 0.95 & 0.95 & 0.96 \\
\hline $\begin{array}{l}\text { Classifier } \\
\text { implementing the } \mathrm{k} \\
\text { nearest neighbors } \\
\text { vote }\end{array}$ & 0.97 & 0.94 & 0.97 & 0.94 & 0.97 & 0.94 & 0.96 & 0.93 \\
\hline $\begin{array}{l}\text { Linear } \\
\text { Discriminant } \\
\text { Analysis }\end{array}$ & 0.73 & 0.89 & 0.73 & 0.89 & 0.73 & 0.89 & 0.73 & 0.89 \\
\hline $\begin{array}{l}\text { Linear Support } \\
\text { Vector } \\
\text { Classification }\end{array}$ & 0.72 & 0.79 & 0.73 & 0.81 & 0.73 & 0.81 & 0.73 & 0.78 \\
\hline $\begin{array}{l}\text { Logistic } \\
\text { Regression aka } \\
\text { logit MaxEnt } \\
\text { classifier }\end{array}$ & 0.75 & 0.97 & 0.76 & 0.97 & 0.76 & 0.97 & 0.75 & 0.96 \\
\hline $\begin{array}{l}\text { Nearest centroid } \\
\text { classifier }\end{array}$ & 0.29 & 0.59 & 0.29 & 0.59 & 0.29 & 0.59 & 0.29 & 0.58 \\
\hline $\begin{array}{l}\text { A random forest } \\
\text { classifier }\end{array}$ & 0.71 & 0.12 & 0.71 & 0.12 & 0.71 & 0.12 & 0.71 & 0.10 \\
\hline $\begin{array}{l}\text { Classifier using } \\
\text { Ridge regression }\end{array}$ & 0.73 & 0.42 & 0.73 & 0.43 & 0.73 & 0.43 & 0.73 & 0.42 \\
\hline $\begin{array}{l}\text { Ridge classifier } \\
\text { with built in cross } \\
\text { validation }\end{array}$ & 0.73 & 0.42 & 0.73 & 0.43 & 0.73 & 0.43 & 0.73 & 0.42 \\
\hline $\begin{array}{l}\text { Gaussian Mixture } \\
\text { Models }\end{array}$ & 0.24 & 0.60 & 0.24 & 0.60 & 0.24 & 0.60 & 0.23 & 0.59 \\
\hline $\begin{array}{l}\text { Support Vector } \\
\text { Machines }\end{array}$ & 0.86 & 0.83 & 0.24 & 0.83 & 0.86 & 0.83 & 0.85 & 0.81 \\
\hline
\end{tabular}


Results of biometrical identification/diagnosis of cardiovascular diseases. Registration time is 10 seconds.

\begin{tabular}{|l|c|c|c|c|c|c|}
\hline \multirow{2}{*}{ Method } & \multicolumn{2}{|c|}{ Haar } & \multicolumn{2}{c|}{ Daubechies } & \multicolumn{2}{c|}{ Symlets } \\
\cline { 2 - 7 } & Diagnosis & Identification & Diagnosis & Identification & Diagnosis & Identification \\
\hline Multiplayer Perception & 0.97 & 0.98 & 0.97 & 0.98 & 0.98 & 0.98 \\
\hline $\begin{array}{l}\text { Naive Bayes classifier for multivariate } \\
\text { Bernoulli models }\end{array}$ & 0.18 & 0.59 & 0.18 & 0.59 & 0.18 & 0.58 \\
\hline A decision tree classifier & 0.89 & 0.82 & 0.89 & 0.82 & 0.89 & 0.81 \\
\hline An extremely randomized tree classifier & 0.97 & 0.97 & 0.97 & 0.97 & 0.97 & 0.97 \\
\hline $\begin{array}{l}\text { Classifier implementing the k nearest } \\
\text { neighbors vote }\end{array}$ & 0.99 & 0.97 & 0.99 & 0.97 & 0.99 & 0.97 \\
\hline Linear Discriminant Analysis & 0.74 & 0.93 & 0.74 & 0.93 & 0.74 & 0.93 \\
\hline Linear Support Vector Classification & 0.72 & 0.88 & 0.72 & 0.88 & 0.73 & 0.89 \\
\hline $\begin{array}{l}\text { Logistic Regression aka logit MaxEnt } \\
\text { classifier }\end{array}$ & 0.76 & 0.97 & 0.76 & 0.97 & 0.76 & 0.97 \\
\hline Nearest centroid classifier & 0.26 & 0.60 & 0.26 & 0.60 & 0.26 & 0.60 \\
\hline A random forest classifier & 0.69 & 0.14 & 0.69 & 0.14 & 0.69 & 0.14 \\
\hline Classifier using Ridge regression & 0.71 & 0.47 & 0.71 & 0.47 & 0.71 & 0.47 \\
\hline $\begin{array}{l}\text { Ridge classifier with built in cross } \\
\text { validation }\end{array}$ & 0.71 & 0.47 & 0.71 & 0.47 & 0.71 & 0.47 \\
\hline Gaussian Mixture Models & 0.24 & 0.74 & 0.24 & 0.74 & 0.24 & 0.74 \\
\hline Support Vector Machines & 0.92 & 0.97 & 0.92 & 0.97 & 0.92 & 0.97 \\
\hline
\end{tabular}


TABLE II (CONTINUE)

Results of biometrical identification/diagnosis of cardiovascular diseases. Registration time is 10 seconds.

\begin{tabular}{|c|c|c|c|c|c|c|c|c|}
\hline \multirow[t]{2}{*}{ Method } & \multicolumn{2}{|c|}{ Coiflets } & \multicolumn{2}{|c|}{ Biorthogonal } & \multicolumn{2}{|c|}{ Reverse biorthogonal } & \multicolumn{2}{|c|}{ Discrete Meyer } \\
\hline & Diagnosis & Identification & Diagnosis & Identification & Diagnosis & Identification & Diagnosis & Identification \\
\hline $\begin{array}{l}\text { Multiplayer } \\
\text { Perception }\end{array}$ & 0.98 & 0.98 & 0.98 & 0.98 & 0.98 & 0.98 & 0.98 & 0.98 \\
\hline $\begin{array}{l}\text { Naive Bayes } \\
\text { classifier for } \\
\text { multivariate } \\
\text { Bernoulli models }\end{array}$ & 0.18 & 0.59 & 0.18 & 0.59 & 0.18 & 0.59 & 0.17 & 0.57 \\
\hline $\begin{array}{l}\text { A decision tree } \\
\text { classifier }\end{array}$ & 0.90 & 0.79 & 0.89 & 0.82 & 0.89 & 0.82 & 0.90 & 0.80 \\
\hline $\begin{array}{l}\text { An extremely } \\
\text { randomized tree } \\
\text { classifier }\end{array}$ & 0.97 & 0.97 & 0.97 & 0.97 & 0.97 & 0.97 & 0.97 & 0.97 \\
\hline $\begin{array}{l}\text { Classifier } \\
\text { implementing the } \mathrm{k} \\
\text { nearest neighbors } \\
\text { vote }\end{array}$ & 0.99 & 0.97 & 0.99 & 0.97 & 0.99 & 0.97 & 0.99 & 0.97 \\
\hline $\begin{array}{l}\text { Linear } \\
\text { Discriminant } \\
\text { Analysis }\end{array}$ & 0.74 & 0.93 & 0.74 & 0.93 & 0.74 & 0.93 & 0.74 & 0.93 \\
\hline $\begin{array}{l}\text { Linear Support } \\
\text { Vector } \\
\text { Classification }\end{array}$ & 0.74 & 0.88 & 0.72 & 0.88 & 0.72 & 0.88 & 0.72 & 0.88 \\
\hline $\begin{array}{l}\text { Logistic } \\
\text { Regression aka } \\
\text { logit MaxEnt } \\
\text { classifier }\end{array}$ & 0.76 & 0.97 & 0.76 & 0.97 & 0.76 & 0.97 & 0.77 & 0.97 \\
\hline $\begin{array}{l}\text { Nearest centroid } \\
\text { classifier }\end{array}$ & 0.27 & 0.60 & 0.26 & 0.60 & 0.26 & 0.60 & 0.26 & 0.59 \\
\hline $\begin{array}{l}\text { A random forest } \\
\text { classifier }\end{array}$ & 0.69 & 0.13 & 0.69 & 0.14 & 0.69 & 0.14 & 0.69 & 0.14 \\
\hline $\begin{array}{l}\text { Classifier using } \\
\text { Ridge regression }\end{array}$ & 0.71 & 0.47 & 0.71 & 0.47 & 0.71 & 0.47 & 0.71 & 0.47 \\
\hline $\begin{array}{l}\text { Ridge classifier } \\
\text { with built in cross } \\
\text { validation }\end{array}$ & 0.71 & 0.47 & 0.71 & 0.47 & 0.71 & 0.47 & 0.71 & 0.47 \\
\hline $\begin{array}{l}\text { Gaussian Mixture } \\
\text { Models }\end{array}$ & 0.24 & 0.74 & 0.24 & 0.74 & 0.24 & 0.74 & 0.22 & 0.73 \\
\hline $\begin{array}{l}\text { Support Vector } \\
\text { Machines }\end{array}$ & 0.92 & 0.97 & 0.92 & 0.97 & 0.92 & 0.97 & 0.91 & 0.96 \\
\hline
\end{tabular}


TABLE III

Results of biometrical identification/diagnosis of cardiovascular diseases. Registration time is 15 seconds.

\begin{tabular}{|c|c|c|c|c|c|c|}
\hline \multirow[t]{2}{*}{ Method } & \multicolumn{2}{|c|}{ Haar } & \multicolumn{2}{|c|}{ Daubechies } & \multicolumn{2}{|c|}{ Symlets } \\
\hline & Diagnosis & Identification & Diagnosis & Identification & Diagnosis & Identification \\
\hline Multiplayer Perception & 0.98 & 0.99 & 0.98 & 0.99 & 0.98 & 0.98 \\
\hline $\begin{array}{l}\text { Naive Bayes classifier for multivariate } \\
\text { Bernoulli models }\end{array}$ & 0.20 & 0.60 & 0.20 & 0.60 & 0.20 & 0.60 \\
\hline A decision tree classifier & 0.91 & 0.84 & 0.91 & 0.84 & 0.91 & 0.83 \\
\hline An extremely randomized tree classifier & 0.98 & 0.98 & 0.98 & 0.98 & 0.98 & 0.98 \\
\hline $\begin{array}{l}\text { Classifier implementing the } \mathrm{k} \text { nearest } \\
\text { neighbors vote }\end{array}$ & 0.99 & 0.98 & 0.99 & 0.98 & 0.99 & 0.98 \\
\hline Linear Discriminant Analysis & 0.74 & 0.93 & 0.74 & 0.93 & 0.74 & 0.93 \\
\hline Linear Support Vector Classification & 0.75 & 0.89 & 0.75 & 0.89 & 0.72 & 0.89 \\
\hline $\begin{array}{l}\text { Logistic Regression aka logit MaxEnt } \\
\text { classifier }\end{array}$ & 0.78 & 0.98 & 0.78 & 0.98 & 0.78 & 0.98 \\
\hline Nearest centroid classifier & 0.28 & 0.63 & 0.28 & 0.63 & 0.28 & 0.63 \\
\hline A random forest classifier & 0.69 & 0.12 & 0.69 & 0.12 & 0.69 & 0.11 \\
\hline Classifier using Ridge regression & 0.71 & 0.44 & 0.71 & 0.44 & 0.71 & 0.44 \\
\hline $\begin{array}{l}\text { Ridge classifier with built in cross } \\
\text { validation }\end{array}$ & 0.71 & 0.44 & 0.71 & 0.44 & 0.71 & 0.44 \\
\hline Gaussian Mixture Models & 0.23 & 0.78 & 0.23 & 0.78 & 0.23 & 0.78 \\
\hline Support Vector Machines & 0.95 & 0.97 & 0.95 & 0.97 & 0.95 & 0.97 \\
\hline
\end{tabular}


TABLE III (CONTINUE)

Results of biometrical identification/diagnosis of cardiovascular diseases. Registration time is 15 seconds.

\begin{tabular}{|c|c|c|c|c|c|c|c|c|}
\hline \multirow[t]{2}{*}{ Method } & \multicolumn{2}{|c|}{ Coiflets } & \multicolumn{2}{|c|}{ Biorthogonal } & \multicolumn{2}{|c|}{ Reverse biorthogonal } & \multicolumn{2}{|c|}{ Discrete Meyer } \\
\hline & Diagnosis & Identification & Diagnosis & Identification & Diagnosis & Identification & Diagnosis & Identification \\
\hline $\begin{array}{l}\text { Multiplayer } \\
\text { Perception }\end{array}$ & 0.98 & 0.99 & 0.98 & 0.99 & 0.98 & 0.99 & 0.98 & 0.98 \\
\hline $\begin{array}{l}\text { Naive Bayes } \\
\text { classifier for } \\
\text { multivariate } \\
\text { Bernoulli models }\end{array}$ & 0.20 & 0.60 & 0.20 & 0.60 & 0.20 & 0.60 & 0.19 & 0.58 \\
\hline $\begin{array}{l}\text { A decision tree } \\
\text { classifier }\end{array}$ & 0.90 & 0.84 & 0.91 & 0.84 & 0.91 & 0.84 & 0.90 & 0.83 \\
\hline $\begin{array}{l}\text { An extremely } \\
\text { randomized tree } \\
\text { classifier }\end{array}$ & 0.97 & 0.97 & 0.98 & 0.98 & 0.98 & 0.98 & 0.98 & 0.98 \\
\hline $\begin{array}{l}\text { Classifier } \\
\text { implementing the } \mathrm{k} \\
\text { nearest neighbors } \\
\text { vote }\end{array}$ & 0.99 & 0.98 & 0.99 & 0.98 & 0.99 & 0.98 & 0.99 & 0.97 \\
\hline $\begin{array}{l}\text { Linear } \\
\text { Discriminant } \\
\text { Analysis }\end{array}$ & 0.74 & 0.93 & 0.74 & 0.93 & 0.74 & 0.93 & 0.74 & 0.93 \\
\hline $\begin{array}{l}\text { Linear Support } \\
\text { Vector } \\
\text { Classification } \\
\end{array}$ & 0.72 & 0.90 & 0.75 & 0.89 & 0.75 & 0.89 & 0.73 & 0.89 \\
\hline $\begin{array}{l}\text { Logistic } \\
\text { Regression aka } \\
\text { logit MaxEnt } \\
\text { classifier }\end{array}$ & 0.78 & 0.98 & 0.78 & 0.98 & 0.78 & 0.98 & 0.78 & 0.97 \\
\hline $\begin{array}{l}\text { Nearest centroid } \\
\text { classifier }\end{array}$ & 0.28 & 0.63 & 0.28 & 0.63 & 0.28 & 0.63 & 0.28 & 0.62 \\
\hline $\begin{array}{l}\text { A random forest } \\
\text { classifier }\end{array}$ & 0.69 & 0.13 & 0.69 & 0.12 & 0.69 & 0.12 & 0.69 & 0.11 \\
\hline $\begin{array}{l}\text { Classifier using } \\
\text { Ridge regression }\end{array}$ & 0.71 & 0.44 & 0.71 & 0.44 & 0.71 & 0.44 & 0.71 & 0.44 \\
\hline $\begin{array}{l}\text { Ridge classifier } \\
\text { with built in cross } \\
\text { validation }\end{array}$ & 0.71 & 0.44 & 0.71 & 0.44 & 0.71 & 0.44 & 0.71 & 0.44 \\
\hline $\begin{array}{l}\text { Gaussian Mixture } \\
\text { Models }\end{array}$ & 0.23 & 0.78 & 0.23 & 0.78 & 0.23 & 0.78 & 0.22 & 0.77 \\
\hline $\begin{array}{l}\text { Support Vector } \\
\text { Machines }\end{array}$ & 0.95 & 0.97 & 0.95 & 0.97 & 0.95 & 0.97 & 0.94 & 0.97 \\
\hline
\end{tabular}


TABLE IV

Results of biometrical identification/diagnosis of cardiovascular diseases. Registration time is 20 seconds.

\begin{tabular}{|c|c|c|c|c|c|c|}
\hline \multirow[t]{2}{*}{ Method } & \multicolumn{2}{|c|}{ Haar } & \multicolumn{2}{|c|}{ Daubechies } & \multicolumn{2}{|c|}{ Symlets } \\
\hline & Diagnosis & Identification & Diagnosis & Identification & Diagnosis & Identification \\
\hline Multiplayer Perception & 0.99 & 0.99 & 0.98 & 0.99 & 0.98 & 0.99 \\
\hline $\begin{array}{l}\text { Naive Bayes classifier for multivariate } \\
\text { Bernoulli models }\end{array}$ & 0.21 & 0.62 & 0.21 & 0.62 & 0.21 & 0.62 \\
\hline A decision tree classifier & 0.92 & 0.85 & 0.92 & 0.85 & 0.92 & 0.86 \\
\hline An extremely randomized tree classifier & 0.98 & 0.98 & 0.98 & 0.98 & 0.97 & 0.98 \\
\hline $\begin{array}{l}\text { Classifier implementing the } \mathrm{k} \text { nearest } \\
\text { neighbors vote }\end{array}$ & 0.99 & 0.98 & 0.99 & 0.98 & 0.99 & 0.98 \\
\hline Linear Discriminant Analysis & 0.73 & 0.94 & 0.73 & 0.94 & 0.73 & 0.94 \\
\hline Linear Support Vector Classification & 0.71 & 0.92 & 0.71 & 0.92 & 0.71 & 0.92 \\
\hline $\begin{array}{l}\text { Logistic Regression aka logit MaxEnt } \\
\text { classifier }\end{array}$ & 0.76 & 0.98 & 0.76 & 0.98 & 0.76 & 0.98 \\
\hline Nearest centroid classifier & 0.31 & 0.64 & 0.31 & 0.64 & 0.31 & 0.64 \\
\hline A random forest classifier & 0.68 & 0.15 & 0.68 & 0.15 & 0.68 & 0.14 \\
\hline Classifier using Ridge regression & 0.69 & 0.45 & 0.69 & 0.45 & 0.69 & 0.45 \\
\hline $\begin{array}{l}\text { Ridge classifier with built in cross } \\
\text { validation }\end{array}$ & 0.69 & 0.47 & 0.69 & 0.47 & 0.69 & 0.47 \\
\hline Gaussian Mixture Models & 0.26 & 0.79 & 0.26 & 0.79 & 0.26 & 0.79 \\
\hline Support Vector Machines & 0.96 & 0.98 & 0.96 & 0.98 & 0.96 & 0.98 \\
\hline
\end{tabular}


TABLE IV (CONTINUE)

Results of biometrical identification/diagnosis of cardiovascular diseases. Registration time is 20 seconds.

\begin{tabular}{|c|c|c|c|c|c|c|c|c|}
\hline \multirow[t]{2}{*}{ Method } & \multicolumn{2}{|c|}{ Coiflets } & \multicolumn{2}{|c|}{ Biorthogonal } & \multicolumn{2}{|c|}{ Reverse biorthogonal } & \multicolumn{2}{|c|}{ Discrete Meyer } \\
\hline & Diagnosis & Identification & Diagnosis & Identification & Diagnosis & Identification & Diagnosis & Identification \\
\hline $\begin{array}{l}\text { Multiplayer } \\
\text { Perception }\end{array}$ & 0.99 & 0.99 & 0.99 & 0.99 & 0.99 & 0.99 & 0.98 & 0.99 \\
\hline $\begin{array}{l}\text { Naive Bayes } \\
\text { classifier for } \\
\text { multivariate } \\
\text { Bernoulli models }\end{array}$ & 0.21 & 0.62 & 0.21 & 0.62 & 0.21 & 0.62 & 0.19 & 0.60 \\
\hline $\begin{array}{l}\text { A decision tree } \\
\text { classifier }\end{array}$ & 0.92 & 0.86 & 0.92 & 0.85 & 0.92 & 0.85 & 0.92 & 0.87 \\
\hline $\begin{array}{l}\text { An extremely } \\
\text { randomized tree } \\
\text { classifier }\end{array}$ & 0.98 & 0.98 & 0.98 & 0.98 & 0.98 & 0.98 & 0.98 & 0.98 \\
\hline $\begin{array}{l}\text { Classifier } \\
\text { implementing the } \mathrm{k} \\
\text { nearest neighbors } \\
\text { vote }\end{array}$ & 0.99 & 0.98 & 0.99 & 0.98 & 0.99 & 0.98 & 0.99 & 0.98 \\
\hline $\begin{array}{l}\text { Linear } \\
\text { Discriminant } \\
\text { Analysis }\end{array}$ & 0.73 & 0.94 & 0.73 & 0.94 & 0.73 & 0.94 & 0.73 & 0.94 \\
\hline $\begin{array}{l}\text { Linear Support } \\
\text { Vector } \\
\text { Classification }\end{array}$ & 0.72 & 0.92 & 0.71 & 0.92 & 0.71 & 0.92 & 0.71 & 0.91 \\
\hline $\begin{array}{l}\text { Logistic Regression } \\
\text { aka logit MaxEnt } \\
\text { classifier }\end{array}$ & 0.76 & 0.98 & 0.76 & 0.98 & 0.76 & 0.98 & 0.76 & 0.98 \\
\hline $\begin{array}{l}\text { Nearest centroid } \\
\text { classifier }\end{array}$ & 0.31 & 0.64 & 0.31 & 0.64 & 0.31 & 0.64 & 0.30 & 0.63 \\
\hline $\begin{array}{l}\text { A random forest } \\
\text { classifier }\end{array}$ & 0.68 & 0.15 & 0.68 & 0.15 & 0.68 & 0.15 & 0.68 & 0.13 \\
\hline $\begin{array}{l}\text { Classifier using } \\
\text { Ridge regression }\end{array}$ & 0.69 & 0.45 & 0.69 & 0.45 & 0.69 & 0.45 & 0.69 & 0.45 \\
\hline $\begin{array}{l}\text { Ridge classifier } \\
\text { with built in cross } \\
\text { validation }\end{array}$ & 0.69 & 0.47 & 0.69 & 0.47 & 0.69 & 0.47 & 0.69 & 0.47 \\
\hline $\begin{array}{l}\text { Gaussian Mixture } \\
\text { Models }\end{array}$ & 0.26 & 0.79 & 0.26 & 0.79 & 0.26 & 0.79 & 0.24 & 0.79 \\
\hline $\begin{array}{l}\text { Support Vector } \\
\text { Machines }\end{array}$ & 0.96 & 0.98 & 0.96 & 0.98 & 0.96 & 0.98 & 0.95 & 0.98 \\
\hline
\end{tabular}

\section{ACKNOWLEDGMENTS}

The reported study was funded by RFBR according to the research project № 19-07-00780

\section{REFERENCES}

[1] S.K. Berkaya, A.K. Uysal, E.S. Gunal, S. Ergin, S. Gunal, and M.B. Gulmezoglu. "A survey on ECG analysis." Biomedical Signal Processing and Control. vol. 43, pp. 216-235. 2018.
[2] M. Merone, P. Soda, M. Sansone and C.Sansone. "ECG databases for biometric systems: a systematic review". Expert Syst. Appl. vol. 67. pp.189-202. 2017.

[3] 3. F.A. Elhaj, N. Salim, A.R. Harris, T.T. Swee, T. Ahmed. "Arrhythmia recognition and classification using combined linear and nonlinear features of ECG signals". Comput. Methods Programs Biomed. vol.127. pp. 52-63. 2016

[4] 4. G.M. Friesen, T.C. Jannett , M.A.Jadallah, S.L. Yates, S.R. Quint , H.T. Nagle. "A comparison of the noise sensitivity of 9 QRS detection algorithms". IEEE Trans. Biomed. Eng. vol. 37. pp. 85-98. 1990 . 
[5] 5. G.D. Clifford, F. Azuaje, P.E. McSharry. Advanced Methods and Tools for ECG Data Analysis, Artech House Publishers, 2006.

[6] 6. P. Chazal, M. O'Dwyer, R.B. Reilly. "Automatic classification of heartbeats using ECG morphology and heartbeat interval features". IEEE Trans. Biomed. Eng. vol. 51.pp. 1196-1206. 2004.

[7] 7. T. Ince, S. Kiranyaz, M. Gabbouj. "A generic and robust system for automated patient-specific classification of ECG signals". IEEE Trans Biomed. Eng. vol. 56. pp. 1415-1426. 2009.

[8] 8. E. Castillo, D.P. Morales, G. Botella, A. Garcia , Parrilla A., A.J. Palma. "Efficient wavelet-based ECG processing for single-lead FHR extraction”. Digit. Signal Process. vol. 23. pp. 1897-1909. 2013.

[9] 9. A. Daamouche , L. Hamami , N. Alajlan, F. Melgani "A wavelet optimization approach for ECG signal classification”, Biomed. Signal Process. vol. 7. pp. 342-349. 2012.

[10] 10. Q. Liu , D. Pitt , X. Wu. "On the prediction of claim duration for income protection insurance policyholders". Ann. Actuarial Sci. vol. 8. pp. 42-62. 2014.

[11] 11. D.P. Coutinho , H. Silva , H. Gamboa , A. Fred, M. Figueiredo. "Novel fiducial and non-fiducial approaches to electrocardiogrambased biometric systems" IET Biometrics. vol. 2. pp. 64-75. 2013.

[12] 12. F.A. Elhaj, N. Salim, A.R. Harris, T.T. Swee, T. Ahmed."Arrhythmia recognition and classification using combined linear and nonlinear features of ECG signals". Comput. Methods Programs Biomed. vol. 127. pp. 52-63. 2016.

[13] 13. M. Seera , C.P. Lim , W.S. Liew , E. Lim , C.K. Loo "Classification of electrocardiogram and auscultatory blood pressure signals using machine learning models". Expert Syst. Appl. vol. 42. pp. 3643-3652. 2015
[14] 14. R.J. Martis, U.R. Acharya, H. Prasad , C.K. Chua, C.M. Lim , J.S. Suri "Application of higher order statistics for atrial arrhythmia classification". Biomed. Signal Process. vol. 8 pp. 888-900. 2013.

[15] 15. D.L. Poole, A.K. Mackworth. Artificial Intelligence Foundations of Computational Agents, Cambridge University Press, 2010

[16] 16. Y. Ozbay, G. Tezel. "A new method for classification of ECG arrhythmias using neural network with adaptive activation function", Digit. Signal Process. vol. 20 pp. 1040-1049. 2010.

[17] 17. T. Ince, S. Kiranyaz, M. Gabbouj. "A generic and robust system for automated patient-specific classification of ECG signals", IEEE Trans Biomed. Eng. vol. 56 pp. 1415-1426. 2009.

[18] 18. S. Wahabi, S. Pouryayevali, S. Hari, D. Hatzinakos. "On evaluating ECG biometric systems: session-dependence and body posture". IEEE Trans. Inf. Forensics Secur. vol. 9. pp. 2002-2013. 2014.

[19] 19. Z. Long, G. Liu, X.Dai. "Extracting emotional features from ECG by using Wavelet transform", in: International Conference on Biomedical Engineering and Computer Science (ICBECS), IEEE, pp. $1-4.2010$

[20] 20. R. Bousseljot, D. Kreiseler, A. Schnabel, "Nutzung der EKGSignaldatenbank CARDIODAT der PTB über das Internet". Biomedizinische Technik, vol. 40, N. 1. p.317. 1995.

[21] 21. The WFDB Software Package. https://physionet.org/physiotools/wfdb.shtml

[22] 22. BioSPPy - Biosignal Processing in Python. https://pypi.org/project/biosppy/ 